\title{
Los surfistas españoles desde un enfoque tribal: aproximación a su descripción como tribu urbana
}

\author{
Paloma Sanz-Marcos ${ }^{1}$ y Dunia Alonso-Sobrado²
}

${ }^{1}$ Universidad de Sevilla y ${ }^{2}$ Universidad de Huelva y Universidad Pablo de Olavide.

Email: ${ }^{1}$ palsanzmarcos@yahoo.es

RESUMEN: El objetivo del presente estudio fue analizar si los practicantes de una determinada actividad deportiva como es el surf en España constituyen una tribu urbana. Para ello, se realizó una descripción de los rasgos que caracterizan a estos surfistas con objeto de actualizar y ofrecer un marco teórico renovado acerca de su caracterización y conceptualización. Se ha opt ado por una metodología cualitativa centrada en los aspectos micro y de significado de las realidades sociales de estos grupos. Como principal técnica de producción de datos se ha empleado el focus group. En concreto, se han configurado cuatro grupos en los enclaves donde esta práctica deportiva cuenta con mayor protagonismo en España: País Vasco, Galicia, Asturias y Andalucía, con un total de 29 participantes. Los resultados fueron similares en todos los grupos, concluyendo que los surfistas cuentan con las mismas características de una tribu urbana, generando así una práctica deportiva vinculada a un sentimiento de pertenencia que va más allá de la identidad individual y deportiva.

PALABRAS CLAVE: surf, práctica deportiva, tribu urbana, identidad colectiva.

\section{Spanish surfers from a tribal approach: Approach to its description as an urban tribe}

ABSTRACT: The objective of the present study was to analyze if the practitioners of a certain sport activity such as surfing in Spain constitute an urban tribe. To do this, it was done a description of the features that characterize these surfers in order to update and offer a renewed theoretical framework about its characterization and conceptualization. A qualitative methodology was used to focus on the micro and meaning aspects of the social realities of these groups. The focus group was used as the main data production technique. Specifically, four groups have been set up in the enclaves where this sport has a greater role in Spain: the Basque Country, Galicia, Asturias and Andalusia, with a total of 29 participants. The results were similar in all the groups, concluding that the surfers have the same characteristics of an urban tribe, generating a sport practice linked to a feeling of belonging that goes beyond the individual and the sporting identity.

KEY WORDS: surfing, sports practice, urban tribe, collective identity. 


\section{INTRODUCCIÓN}

El concepto de tribu urbana fue acuñado por el sociólogo francés Michel Maffesoli quien en 1990 utilizó el vocablo tribu para explicar una realidad propia de la sociedad posmoderna que, contextualizada en un regreso paulatino hacia estructuras arcaicas, se materializaba en un proceso de gestación de una serie de vínculos grupales. Maffesoli recupera un término que remite a lo primitivo, a lo arcaico, a aquello que se antepone a lo civilizatorio y moderno. Autores como Feixa (1999) entienden que existe una presencia de lo étnico o rural en las culturas contemporáneas y, en especial, en las culturas juveniles. A tales efectos, asegura que "entre indígenas y jóvenes hay notables paralelismos: ambos son grupos subalternos, tutelados por el Estado, tachados de inmaduros e infantilizados; ambos se organizan en estructuras comunitarias, lucen atuendos y lenguajes exóticos, son dados a la fiesta y al ritual" (Feixa, 1999, p.137).

Esta investigación se centra en el caso concreto de la tribu de los surfistas (Lefineau, 2010). El surf, en su sentido más general, consiste en el acto de mantenerse en pie encima de una tabla y guiarla a través de la superficie de la cara de una ola rompiente (Booth, 1999). Esta actividad cuyo origen se remonta a finales del siglo XIX, ha suscitado a lo largo de los últimos años un gran interés por parte de la sociedad española que ha visto crecer el número de practicantes de forma exponencial en los últimos años. Según los datos más recientes disponibles a través del Anuario de Estadísticas Deportivas de 2018 elaborado por el Ministerio de Educación Cultura y Deporte, en España hay en la actualidad: 30.555 personas federadas, 164 clubes y se organizan un total de 105 competiciones anuales. Asimismo, el reflejo de los datos anteriormente expuestos y la oportunidad de practicar este deporte en nuestro país -España cuenta con casi 8.000 kilómetros de costa-, subrayan la importancia potencial de este deporte.

Dado que la literatura académica disponible acerca de esta tribu ofrece una visión subjetiva y estereotipada de lo que identifica a los surfistas (Colubi, 1997; Caffarelli, 2008; Lefineau, 2010), el principal objetivo que persigue esta investigación es describir los rasgos que caracterizan a estos surfistas como tribu urbana con objeto de actualizar y ofrecer un marco teórico renovado acerca de su caracterización, descripción y conceptualización con el fin de poder actualizar su conceptualización para futuras investigaciones vinculadas a otras disciplinas.

\subsection{La tribu urbana}

Encontrar una única definición de tribu urbana se antoja un proceso variable y huidizo, difícil de objetivar en un cuadro fijo y definitivo (Costa, Pérez y Tropea, 1996, p.93). Las tribus urbanas resultan ser complejas, de forma que existen múltiples maneras y diferentes disciplinas desde las que abordarlas (Fernández, 2000, p.270). No obstante, el concepto posee una identidad propia evidenciada a partir de una serie de características que contribuyen a su conceptualización y descripción. Estos rasgos son, su vinculación con la juventud, una concepción concreta del espacio y el tiempo, la ejecución de una serie de rituales y la caracterización de sus miembros mediante una serie de rasgos identificativos.

Al vincular la tribu con la juventud es necesario recordar que las etapas de la vida tienen un origen no solo natural, sino también social. Con objeto de construir la identidad del individuo, esta etapa supone para el mismo la construcción de una unión en diferentes tipos de agrupamientos que, a su vez, conllevan a la constitución de una 
"familia en transición" que facilita su desarrollo en sociedad (Caffarelli, 2008, p.36). En relación al concepto de juventud, y sin entrar en las definiciones de los conceptos sociológicos de "generación", tratado en sus inicios por Karl Mannheim, y el de "etapas de la vida", sí destacamos lo señalado por Martín Criado en cuanto a lo considerado por Mannheim en relación al concepto de generación y a las diferentes condiciones sociales y materiales que implica en el proceso de socialización:

Estas variaciones cobran relevancia por la importancia de las primeras etapas de la vida en la producción del individuo: en la 'estratificación de la experiencia': su formación supone unas determinaciones negativas y positivas sobre lo perceptible, lo pensable, lo factible. (1998, p.81)

En este sentido, hay que tener en cuenta también el concepto de habitus de Bourdieu (1998) cuando tratamos el concepto de juventud, ya que no son grupos uniformes, sino que van asociados al contexto social de cada individuo.

A tales efectos, las tribus urbanas se erigen como agrupaciones que ayudan al proceso de socialización del individuo. Siguiendo a Greco (2003), se entiende por socialización una forma en la que cada individuo se incorpora a la sociedad y a su vez, al conjunto de mecanismos mediante los cuales los individuos realizan el aprendizaje de las relaciones sociales y asimilan las normas, los valores y las creencias de esa sociedad o colectividad (Greco, 2003). Se trata, por tanto, de un proceso que no solo permite el acceso a la sociedad, sino la integración del individuo en ésta a través de la comprensión de ciertas normas, valores y creencias. En consecuencia, en la pertenencia a las tribus, el individuo adquiere consciencia de su identidad. De hecho, las tribus urbanas representan un instrumento del que se valen los jóvenes para buscar su identidad, elaborar su imagen social y la forma en que se muestran ante los demás (Caffarelli, 2008, p.52).

A este parecer, Tajfel (1978) señala que los grupos sociales son una fuente de identidad para el sujeto, esto es, proporcionan identidad a sus miembros. Así lo entienden Turner y Brown (1978) cuando, basándose en las teorías preliminares de Tajfel, afirman que los individuos se definen y evalúan en cuanto a sus grupos sociales y que son éstos los encargados de aportar identidad social para sus miembros. En efecto, esas evaluaciones merecen especial atención en este contexto, ya que la identidad de los miembros está vinculada a, por un lado, la conciencia que tienen los mismos como miembros del propio grupo y, por otro, a toda la significación emocional que supone esa pertenencia, entendiendo esta significación emocional como la evaluación que los sujetos hacen de sí mismos cuando están dentro de un grupo. Como Tajfel (1978) asegura, la pertenencia de un individuo a un grupo contribuye de forma positiva o negativa a la imagen que éste tiene de sí mismo. Por lo tanto, y como sostienen Turner y Brown (1978), la pertenencia a un grupo influye en la creación de la identidad de sus miembros. Por su parte, Vargas señala que en la configuración de la identidad del individuo se relaciona con el modo en el que las experiencias mutuas de los jóvenes se expresan socialmente mediante la construcción de estilos de vida propios y distintivos (2012, p.149). En este sentido, si la identidad alude a la toma de consciencia de un grupo cuando se enfrenta a otro, a la visibilización de los contrastes socioculturales, se podría afirmar que se produce lo que Márquez Lepe denomina "culturalización de las diferencias y una naturalización de las mismas (2015, p. 148). En este escenario de generaciones identitarias es donde operan y surgen las tribus.

Otra de las particularidades que definen a las tribus urbanas es su necesidad de establecimiento y organización espacial. La tribu necesita un lugar en el que 
ordenarse, un espacio vital que ayude a que el grupo se cohesione, o como Giner, Lamo de Espinosa y Torres (2006) afirman, las tribus "[...] acotan un espacio [...] porque es indispensable para establecer una red de relaciones que fortalecen el sentimiento de pertenencia al grupo" (p.928). Ganter y Zarzuri (1999) observan que la construcción de esas nuevas relaciones que conforman los jóvenes a través de las tribus urbanas genera "construcciones de identidades asociadas a expresiones particulares o geografías específicas" (p.14). Por su parte, Maffesoli (1990) señala que la existencia de un mismo territorio compartido favorece la idea de comunidad que caracteriza a estas tribus (1990). Los espacios de la tribu suponen lugares de encuentro donde las identidades colectivas tienden a reafirmarse y fijarse, más que a disolverse (Matus, 2000, p. 109). El espacio de la tribu es más distinguible por su condición unificadora que por el establecimiento de un lugar en el que manifestar las diferencias. Costa, Pérez y Tropea añaden que, "el espacio presenta un ámbito para la construcción del discurso de las tribus urbanas" (1996, p.127). Así, las tribus urbanas no solamente ocupan una realidad física-0, dicho de otro modo, topográfica; a su vez, esta ocupación "conlleva intrínsecamente un factor de significación: El «espacio vivencial o vivido»" (Aguirre y Rodríguez, 1997, p.27).

En relación a la importancia del uso simbólico del espacio entre las tribus y, en concreto, en el uso del espacio público en el surf, una de las aportaciones más interesantes en este último año la ha realizado de nuevo Esparza (2019). Este autor afirma que esta práctica deportiva muestra el inicio de "una conquista del uso del espacio público para uso deportivo donde, a diferencia de otros usos deportivos (...) las mujeres tenían que competir y luchar directamente con los hombres" (Esparza, 2019 , p. 73). Una de las implicaciones que el espacio tiene para la organización de la identidad tribal es su característica de escenario para la ejecución de rituales y ceremonias (Silva, 2002, p.123) que tienen una fuerte implicación para la conformación de la identidad de los miembros de la tribu. En efecto, el ritual ofrece una ocasión para cohesionar al grupo y facilitar la incorporación de su sentido de pertenencia. Aguirre y Rodríguez profundizan en esta idea, subrayando que el ritual conlleva una importante responsabilidad, ya que "el grupo necesita de una cultura que vertebre para poder existir, vivenciada a través de unos rituales que mantengan el sentido de pertenencia entre sus integrantes" (1997, p.27). De este modo, el ritual para las tribus asegura el perdurar del grupo en su conjunto (Maffesoli, 1990, p.47). Ciertos eventos, como las citas deportivas, adquieren una importancia vital que representa un "simbolismo, es decir, la impresión de participar en una especie común" (Maffesoli, 1990, p.179).

Asimismo, el conocimiento por parte del individuo de esos rituales y códigos representacionales supone la transgresión de las reglas socialmente instituidas (Ganter y Zarzuri, 1999, p.10). Es por ello que, cuando los miembros "[...] se visten, se adornan o se comportan siguiendo ritos, ritmos y costumbres que no pertenecen a la normalidad adulta, están manifestando su rebeldía y buscando, a través de ella, la construcción de una nueva identidad [...]" (Soto, 2012, p.118).

La pertenencia a la tribu implica una serie de condicionantes estéticos que ofrecen a sus miembros el desarrollo de actitudes y comportamientos comunes a los del resto del grupo, gracias a los cuales dejan de ser sujetos anónimos para pasar a ser considerados como individuos que pertenecen a un colectivo. De este modo, reafirman su identidad, su "ser persona", y su sentido de pertenencia a la agrupación, la cual, asimismo, se refuerza al participar en experiencias conjuntas y rituales; por ejemplo, fiestas, conciertos, etcétera, en los que se pone de manifiesto ese estilo estético (Caffarelli, 2008, p.48). 
Por supuesto, no se puede perder de vista que los propios conceptos de identidad y tribu no dejan de ser conceptos analíticos, que afectan tanto al individuo como a los grupos y que, como tal, están en una constante evolución derivada de su propia instrumentalización. En este sentido, cualquier análisis debe tener en cuenta que tanto las identidades como las tribus están en constante evolución, no son conceptos estáticos, sino que están en continuo proceso de renegociación (Bauman, 2010) por lo que el presente trabajo también contribuye a la actualización de esta constante redefinición.

\subsection{El surf en España}

El surf en España es un deporte que ha venido creciendo en número de practicantes de forma exponencial en los últimos años. Los inicios de este deporte en España han sido ampliamente abordados por Daniel Esparza, experto investigador en torno a la historia del surf en España y creador del centro de investigación de la historia del surf y el bodyboard, conocido como Olo Surf History, quien asegura que "la génesis del surf en España fue bien diferente a cómo ocurrió en otros países [...] no surgió desde un núcleo concreto a partir del cual se expandió por el resto del país ejerciendo influencia sobre el resto [...]" (Esparza, 2016, p.164).

A lo largo del tiempo, el surf español se ha ido consolidando. Entre las zonas más desarrolladas en España destacamos la comunidad autónoma del País Vasco. Además, en esta comunidad autónoma es donde han tenido lugar los primeros campeonatos de surf del país (Esparza, 2016), destacando la playa de Mundaka como el primer lugar en España en el que se han desarrollado pruebas oficiales del circuito profesional mundial del surf.

No obstante, otras zonas reseñables en torno a la práctica del surf en España son Asturias y Cantabria. En esta última, sobresale el núcleo santanderino, el cual "fue el más activo y el que más rápido evolucionó en los primeros años de surf en España" (Esparza, 2016, p.112) y que aún goza de gran popularidad. Por otra parte, destaca en el noroeste de España la región de Galicia, que cuenta con uno de los primeros surfcamps españoles y donde además se celebra actualmente una prueba del circuito de la Liga mundial de surf (WSL ${ }^{1}$ ), el conocido Pantín Surf Classic en la playa de Pantín situada en la zona de Ferrol. Al mismo tiempo, destacan en el sur de España diferentes localizaciones andaluzas: desde el inicio de su costa occidental en Huelva con playas como Mazagón, donde existe una larga tradición del surf, avanzando hacia la costa de Cádiz, pasando por Málaga - pionera en el Mediterráneo (Esparza, 2015) - , llegando finalmente hasta la costa oriental en Almería. Andalucía cuenta con una importante cantidad de surfistas que se reconocen en unos 2000 federados por la Federación Andaluza de Surf.

En un segundo plano, subrayamos la costa Mediterránea que, debido a su menor exposición al mar abierto, cuenta con menor afluencia de condiciones meteorológicas que favorezcan la práctica del surf. Siguiendo a Pellón (2009), destaca la zona de Cataluña, con Barcelona a la cabeza, o las Islas Baleares. Es preciso reconocer asimismo la tradición canaria desde principios de los 60 (Esparza, 2016), debido a que cuenta con unas condiciones meteorológicas excelentes para su práctica, así como algunas de las mejores olas de todo el continente europeo (Pellón, 2009).

En definitiva, es posible reconocer que el surf en España se encuentra entre una de las prácticas deportivas con mayor potencial en cuanto a su proyección

\footnotetext{
${ }^{1}$ Las siglas WSL corresponden a World Surfing League
} 
económica, además de lograr adquirir cada año un mayor número de adeptos. Partiendo de la popularidad y auge de este deporte, esta investigación persigue, en primer lugar, conocer en profundidad a los surfistas españoles. De manera concreta se pretende comprobar si, en efecto, pueden ser considerados como una tribu urbana y, en último término, contribuir a la actualización de la literatura académica al respecto.

\section{MÉTODO}

Para conocer en profundidad a los surfistas españoles y comprobar si, en efecto, pueden ser considerados como una tribu urbana y poder contribuir a la actualización de la literatura académica, se ha llevado a cabo una metodología de tipo cualitativa (focus group o grupo focal).

En primer lugar, se ha llevado a cabo una revisión de la literatura académica disponible en torno a los surfistas españoles. Es preciso destacar que el estudio se ha ocupado de analizar el fenómeno desde el año 1990, primera referencia del concepto de tribu urbana en la literatura académica, hasta la reciente obra de Warren y Gibson (2017) dedicada a la gestión empresarial de la industria del surf. Dicho criterio temporal ha permitido obtener tanto una información transversal del objeto de estudio, como una visión holística del concepto ya que, de otra forma, no se hubiese podido observar la evolución del fenómeno en conjunto con otros términos aparecidos a lo largo del tiempo así como el modo en el que se interrelacionan sus características e implicaciones.

\subsection{Instrumento}

La revisión de la literatura científica ha constituido una primera toma de contacto y punto de partida para conocer e introducir la siguiente y principal técnica de producción de datos de esta investigación, el focus group o grupo focal, con el objeto de conocer en profundidad el comportamiento de los surfistas bajo una óptica social (Rubin y Rubin, 1995; Walle, 2015). Siguiendo a Rubin y Rubin (1995), esta técnica es adecuada para estudiar a los posibles miembros de una comunidad en tanto que los focus groups son una forma de evaluación en la que se concentran grupos de personas para debatir posibles cambios de impresiones. Los asuntos discutidos van desde lo más específico y conciso hasta las preocupaciones más amplias de los miembros de una comunidad, de manera que parece útil para el estudio de la comunidad surfista. Asimismo, los focus groups se pueden describir como un pequeño grupo de personas que han sido elegidas para explorar ciertos temas o preguntas. El método consiste en generar una conversación con la esperanza de que la sinergia del grupo conduzca a nuevas y útiles ideas (Walle, 2015). Guiados por un moderador, los focus groups suponen entrevistas en grupo cuyas conversaciones, generadas por sus participantes, conforman los datos esenciales a recoger para la investigación. A pesar de que existen numerosas variaciones, el número de participantes oscila entre un mínimo de seis y un máximo de diez individuos (Morgan, 1998; Taylor, Bogdan y Devault, 2015). Debido a que el objeto de investigación con pequeños grupos, esta técnica se muestra como una de las más acertadas debido a que reproducen situaciones sociales (Martín Criado, 1997) y nos permite llegar al significado que cada participante da a su discurso.

Para el diseño del contenido de los focus groups, en primer lugar, y siguiendo la lógica científica, se ha partido de los objetivos del estudio para elaborar las preguntas concretas a realizar en la muestra empírica. Cabe destacar que se han operacionalizado las preguntas en función de la literatura académica, teniendo en cuenta las teorías acerca de la tribu urbana y el surf como deporte. En este sentido, aunque se elaboró un primer guion con preguntas, finalmente se optó por elaborar 
diversos bloques temáticos a tratar, que se repitieron en todos los grupos. Sin embargo, la secuencia de preguntas dentro de cada bloque iba variando según la dinámica del propio grupo y a consideración de la investigadora que lo estaba realizando. Los bloques temáticos fueron: identidad y surf, identidad y etapa de la vida (adolescencia, juventud, adultez), estilos de vida y surf, estilos de consumo y práctica deportiva.

\subsection{Participantes}

Para la muestra, se realizó una selección intencional de los sujetos informantes, combinando los procedimientos de selección por conveniencia y, a continuación, la de contactación por bola de nieve. Por conveniencia debido a los contactos personales y deportivos de una de las investigadoras y por bola de nieve debido a la necesidad de completar la muestra necesaria para la realización de los grupos. A todas las personas que participaron se les pasó un acuerdo de confidencialidad, garantizando la anomización de las fuentes. De hecho, para lograr el anonimato, la única codificación de la información en el análisis se estableció asignando un código que identifica al individuo y a su grupo, sin incluir ninguna otra información. En definitiva, y ante posibles críticas a la intencionalidad de la muestra que se emplea, estamos de acuerdo con Martín Criado (1997), quien afirma lo siguiente:

La validez externa de esta técnica la fundamentamos a dos niveles: por un lado, la captación y la dinámica interaccional conforman el grupo de discusión como un dispositivo de homogeneización; por otro, el tipo de diseño y de análisis de discurso nos van a dar las condiciones de generalización del análisis (p.107).

Concretamente, se han llevado a cabo cuatro focus group que han contado con un total de 29 participantes procedentes de aquellas zonas españolas donde la práctica del surf tiene mayor presencia. Esto es, la zona norte occidental de España en La Coruña (ocho participantes), la zona norte central del cantábrico en Asturias (seis participantes), la zona norte más oriental del cantábrico en el País Vasco (siete participantes) y la zona sur con un grupo celebrado en Andalucía (ocho participantes).

En lo relativo a ciertas particularidades de los sujetos, cabe destacar que se ha contado con surfistas experimentados $(n=29)$, el $82,75 \%$ aseguraba llevar más de 10 años practicando surf y las edades comprendidas has oscilado entre los 18 años y los 49 años, situándose entre la treintena la gran mayoría. Se ha intentado representar a la mujer en todos los grupos formados. No obstante, y siguiendo el estudio anterior de Mountinho, Dionísio y Leal publicado en 2007, es complejo encontrar a mujeres que practiquen surf. Es por ello que, entre los cuatro focus groups realizados, en el de la zona de Galicia y Asturias se ha contado con una única mujer respectivamente, en el de la zona de Cádiz con dos mujeres, y en el caso particular de la zona de País Vasco no se pudo contar con la presencia de ninguna mujer, a pesar de provocar su participación. Es preciso destacar que con objeto de facilitar la lectura de los resultados se ha optado por usar los siguientes acrónimos para distinguir a los participantes: PPV, para indicar las respuestas de los participantes del País Vasco, PG7 para indicar las respuestas de los participantes gallegos, PPC para indicar las respuestas de los participantes procedentes de Cádiz y PPA para indicar las respuestas de los participantes asturianos.

\subsection{Procedimiento}


En cuanto al proceso de producción de datos, todas las entrevistas tuvieron lugar durante el mes de enero de 2018. En el caso de los grupos realizados en las zonas de Galicia y Asturias, los encuentros tuvieron lugar en la sala de reuniones de los hoteles NH Coruña Centro en La Coruña el 20 de enero a las 20:00 h. y en el Hotel Carbayón en Oviedo el 21 de enero a las 19:15 h. En cuanto a los grupos llevados a cabo en la zona de País Vasco y Andalucía, se celebraron en centros públicos que fueron cerrados al público para tal ocasión: Club Shelter Surf en el caso de Zarauz el día 23 de enero a las 20:38 h. y en la Asociación de Vecinos de El Palmar en Cádiz a fecha de 27 de enero a las 20:12 h. Cabe destacar que los espacios dieron lugar a una conversación distendida y armoniosa y que se promovió la participación de los sujetos y el bienestar de los mismos, proporcionando agua y comida, tal y como aconseja Morgan (1998, p.128). En este sentido, el transcurso de las entrevistas se desarrolló sin incidencias o problemas que imposibilitaran o condicionaran la conversación. En cuanto a la duración de las entrevistas, ocuparon entre 40 y 96 minutos, siendo la de mayor duración la celebrada en Zarauz con un total de 96 minutos, y la más corta la llevada a cabo en Cádiz con una duración de 40 minutos. Los encuentros fueron celebrados en lugares que permitieran el dinamismo y bienestar de los participantes, en el caso de Galicia y Asturias, los encuentros tuvieron lugar en la sala de reuniones de un hotel y para el País Vasco y Andalucía, se celebraron en centros públicos que fueron cerrados al público para tal ocasión.

\section{4. Análisis de los datos}

Por último, como técnica de análisis se empleó el análisis del discurso, identificando aquellos marcos conceptuales 0 de significado que vinculan las situaciones y relaciones establecidas en estos grupos con las definiciones teóricas de las tribus (Martín Criado, 1997). Estos marcos que se han tratado de hallar han sido los marcos de interpretación de los cuatro bloques temáticos establecidos en los grupos, interpretándolos mediante la vinculación con el objeto principal de este estudio, la identificación o no de los surfistas españoles como tribu urbana a través de los rasgos que los caracterizan. Por tanto, estos marcos los van construyendo los propios participantes de los grupos con su interacción comunicativa, al constituirse como grupo homogéneo por su vínculo con la práctica deportiva del surf.

\section{RESULTADOS Y DISCUSIÓN}

\subsection{Resultados y discusión de la revisión de la literatura}

El análisis de la literatura académica disponible en torno a los surfistas en España se ha presentado escasa. Apenas existen referencias que aborden el comportamiento de este colectivo en nuestro país, así como tampoco abundan las referencias 0 los estudios rigurosos multidisciplinares que describan las particularidades de los surfistas en general. Es por ello que se ha hecho necesario conjugar varias fuentes de información. Por un lado, se han tratado aquellas referencias bibliográficas encontradas en torno al estudio de grupos de surfistas en países como Australia, EE.UU. y Reino Unido. En el análisis de estas referencias, observamos que, a través de una serie de artículos académicos elaborados por expertos con una larga tradición en el estudio del surf, muchas de las particularidades de este grupo de consumidores comparten similitudes con los surfistas españoles. Por otro lado, se ha hecho una revisión de publicaciones españolas especializadas en surf a través de la consulta de revistas como Surfer Rule, Hebe Magazine, Tres60 o RadicalSurf, las cuales han ofrecido ejemplos muy ilustrativos de la realidad española 
en este deporte. Asimismo, se ha contemplado la obra Surfers. Una visión antropológica de la cultura del surf (2006) de Alain Gonfaus, que proporciona un excelente punto de partida para entender de forma directa cómo es la tribu surfista en España. El autor -el cual se propone que: "El objetivo de este libro es ofrecernos la oportunidad de acercarnos a la cultura de la "Tribu del Mar»" (Gonfaus, 2006, p. 5)— ofrece una serie de entrevistas a algunos de los primeros surfistas de las costas vascas - concretamente de la ciudad de San Sebastián- que ponen de manifiesto el sentimiento en primera persona de surfistas asiduos a este deporte. Todos estos testimonios parten de una pregunta inicial: ¿Qué representa el surf para ti a nivel personal? Precisamente, este primer planteamiento sirve como punto de partida para revelar uno de los primeros rasgos de esta tribu. Este monográfico se abre con el testimonio de Iñigo Iraola, uno de los entrevistados por Gonfaus, quien señala que: "Aunque suene a frase hecha, el surf es una forma de vida, y como la vida misma, con el paso de los años va tomando diferentes perspectivas [...]" (Gonfaus, 2006, p.85). Este testimonio plantea que el surf representa algo que supera lo deportivo. En este sentido, el surf no solo ofrece un estilo de vida o una actividad física, sino que encierra una serie de experiencias que según Preston-Whyte (2002) proporcionan al individuo un significado vital (p. 319). De estas manifestaciones se desprende que los surfistas comparten una pasión común hacia la actividad que practican. Así lo entiende Juan Arteche, uno de los primeros surfistas españoles, cuando afirma que: "[...] el surf me fue envolviendo y se fue convirtiendo en una auténtica pasión, en una relación muy intensa, en algunos casos hasta obsesiva [ ...]" (Gonfaus, 2006, p.13). De entre los valores que motivan a los surfistas a practicar la actividad, se ha observado que el argumento relativo a la preocupación por la salud o por mantenerse en forma, no son factores que verdaderamente motiven su práctica. Como expresa Javier Arteche ante la pregunta de qué representa el surf para él: "Aparte de las sensaciones únicas y momentos mágicos e inolvidables que he vivido surfeando y compartiendo con amigos, la pasión por un deporte irrepetible. El surf representó para mí un hito en mi vida" (Gonfaus, 2006, p.12).

Es preciso destacar que entre los surfistas parece que existe una conciencia colectiva puede que se manifiesta agudamente a través del sentimiento de una comunidad en torno a una playa determinada. Es lo que comúnmente se denomina como "localismo" entre los surfistas. Para entender el origen de esta conciencia, es necesario atender a una cuestión fundamental para la práctica de esta actividad. Siguiendo a Stranger, para que las olas sean surfeables, es necesario que se reúnan varios elementos naturales, como grandes marejadas, vientos en alta mar y momentos de mareas correctas. Un surfista necesita una combinación de un estilo de vida flexible y parte de suerte para estar en el lugar correcto en el momento adecuado. Todos estos elementos solo pueden estar en armonía durante una hora o dos antes de que cambien las condiciones, y no volverán a darse hasta dentro de muchas semanas (2010, p.1120).De esta idea se desprende que para la práctica del surf son necesarios unos condicionantes meteorológicos especiales que, como ejemplifica coloquialmente Carlos Ibáñez, pueden suponer que "te pasas el día controlando el mar, que si sube el golpe, que si sale viento, que si en la «plea», que si en la «baja» funciona [...]" (Gonfaus, 2006, p.17).

Asimismo, se observa que existen ciertos rituales que caracterizan a los surfistas y que pueden agruparse en dos tipos diferenciados. Por un lado, encontramos aquellos rituales que contribuyen al óptimo desarrollo del surf y se presentan como imprescindibles para la consecución de su práctica; ejemplo de ello son la colocación del neopreno cuando el clima lo requiere, la preparación de la tabla 
mediante su encerado (Moutinho et al., 2007, p.667), la colocación de quillas ${ }^{2}$ o la disposición del invento o leash. En este sentido, se observa que la tribu consumidora española presenta ciertos patrones de consumo asociados a dichos rituales. La compra del material para la actividad surfista como el neopreno, obedece a una decisión de compra importante para el practicante de surf español. Seleccionar el grosor del mismo, el tipo de material o incluso la marca, son decisiones decisivas para afrontar las condiciones del mar. Por otro lado, y como veníamos comentando, son igualmente frecuentes otros rituales que, si bien contribuyen indirectamente al desarrollo de la actividad, se presentan como acciones habituales generalizadas entre los sujetos. Es el caso de la práctica de evaluación de las condiciones del mar: Los surfistas pueden situarse por un largo periodo de tiempo frente al mar para valorar el estado de las olas con objeto de decidir si llevar a cabo la práctica en ese momento. En este sentido, es común esperar el tiempo suficiente hasta que la serie ${ }^{3}$ llegue a la orilla para determinar su estado (Moutinho et al., 2007, p.677). Otro ejemplo lo encontramos en las relaciones y hábitos que suelen darse entre surfistas mientras practican surf. Las exclamaciones y muestras de admiración y celebración entre surfistas cuando se consigue realizar una buena maniobra o surfear una ola excelente, son asimismo prácticas habituales.

Al mismo tiempo, esta tribu cuenta con una serie de "normas" no regladas oficialmente - salvo en el caso de competiciones-que contribuyen al reconocimiento y descripción de este tipo de tribu. Entre algunas de ellas destacamos la regla de respetar la posición de preferencia en la ola (Anderson, 2013, p.963) la cual se establece como una forma de evitar lo que se entiende por "saltar" la ola o snake the wave en inglés, y que, como indica Booth, se trata de remar la ola en perjuicio del que tiene preferencia (2008, p.17). Estas normas ponen de manifiesto otro componente del capital tribal del grupo. Los surfistas emplean cierto lenguaje distintivo que los diferencia de otras tribus para expresar determinadas ideas. Como afirma Pellón (2009), los surfistas españoles tienen su propio método de medir las olas que difiere "bastante" de los metros o centímetros empleados por el resto de sistemas que habitualmente utilizan los miembros de las tribus surfistas de otros países, tal y como se observa en la Tabla 1.

Tabla 1. Clasificación del tamaño de las olas. Elaboración propia a partir de Pellón (2009)

\begin{tabular}{|c|c|c|}
\hline $\begin{array}{c}\text { Denominación especial de la } \\
\text { ola por parte de la tribu } \\
\text { española }\end{array}$ & $\begin{array}{c}\text { Tamaño "real" de la } \\
\text { ola }\end{array}$ & $\begin{array}{c}\text { Equivalencia con la } \\
\text { denominación estándar } \\
\text { aceptada por el resto de } \\
\text { tribus surfistas }\end{array}$ \\
\hline Ola "pequeña" & $\begin{array}{l}\text { Ola de hasta } 1 \mathrm{~m} \text { de } \\
\text { altura «real» }\end{array}$ & 61 pies \\
\hline Ola de medio metro & $\begin{array}{c}\text { Ola de hasta } 1,5 \mathrm{~m} \text { de } \\
\text { altura }\end{array}$ & 61,5 pies. \\
\hline Ola de un metro & $\begin{array}{c}\text { Ola de hasta } 2 \mathrm{~m} \text { de } \\
\text { altura }\end{array}$ & 62 pies. \\
\hline Metro "pasado" & $\begin{array}{c}\text { Ola que sobrepasa el } \\
\text { metro }\end{array}$ & 63 pies \\
\hline
\end{tabular}

\footnotetext{
${ }^{2}$ Las quillas son una especie de aletas que se colocan en la parte trasera de la tabla y que funcionan a modo de timón para facilitar la dirección y velocidad de la tabla.

${ }^{3}$ Las olas no se encuentran de forma continua en el mar a pesar de que haya oleaje. Es frecuente que se presenten en "series" o bloques de olas que se aprecian en intervalos de segundos. De tal modo que un periodo largo puede ser de unos 14 segundos, indicando éste que cada 14 segundos tendrá lugar la siguiente serie de olas.
} 


\begin{tabular}{c|c|c}
\hline "Metrazo" & $\begin{array}{c}\text { Ola que sobrepasa el } \\
\text { metro, pero } \\
\text { contundente y tubera }\end{array}$ & 63 pies \\
\hline Ola de metro y medio & $\begin{array}{c}\text { Ola de hasta 2,5 m de } \\
\text { altura }\end{array}$ & 66 pies \\
\hline $\begin{array}{c}\text { Ola de dos metros y medio a } \\
\text { tres metros } \\
\begin{array}{c}\text { (A partir de esta medida el } \\
\text { autor destaca que se } \\
\text { denominan como "olas } \\
\text { grandes") }\end{array}\end{array}$ & $\begin{array}{c}\text { Olas de más de } 5 \mathrm{~m} \\
\text { de altura }\end{array}$ & 68 pies \\
\hline
\end{tabular}

Asimismo, la elección de la ropa y la apariencia supone un importante rasgo diferenciador de los surfistas (Irwin, 1973, p.141), el uso de determinadas marcas puede incluso suponer el reflejo del grado de capital tribal incorporado por los miembros de la tribu. De hecho, incluso el punto de venta escogido puede determinar el grado de autenticidad del miembro de la tribu.

\subsection{Resultados y discusión de los focus groups celebrados}

Los resultados de los focus groups manifiestan que el diseño de la entrevista fue adecuado para la obtención de resultados interesantes para la investigación. Las respuestas de los surfistas indican desde un inicio que su consideración como grupo con características similares es posible. Así lo demuestran sujetos como el participante PPV1 quien afirma que: "Prefiero surfear con amigos olas peores, que olas muy buenas solo" o el sujeto PPV3 quien puntualiza que, de hecho, practicarlo solo le parece algo aburrido cuando asegura que "yo haciéndolo solo me aburro". De esta manera, se observa que tanto la compañía de calidad como el número de personas que conforman dicha compañía es algo muy valorado entre los surfistas. Otra de las tendencias observadas en esta línea es la destacar que la presencia de amigos se observa favorable debido a que refuerza la autoestima de los surfistas. Es el caso que apunta el PG7 quien señala que: "[...] siempre te apetece si coges una ola buena que alguien te la vea, un amigo".

Las respuestas de los surfistas han resultado reveladoras en torno a la descripción de un estilo identificable. Diversos participantes reconocen la existencia de un estilo característico entre aquellos que surfean. Se trata de opiniones como la del PPA7 quien señala que "[...] todos más o menos tienen una estética relativamente parecida, aunque nosotros nos notamos muy diferentes seguramente cuando alguien nos vea desde fuera, de aquí, les parezcamos relativamente iguales". De esta manera se perciben por los individuos las identidades propias de un grupo frente a otro. De un modo similar, el PPG9 añade que: "Yo creo que hay distintos estilos, pero no hay un estilo que sea propio de un surfista, normalmente el surfista suele tener flow ${ }^{5}$, y suele tener estilo, yo creo". De hecho, la PPG5 señala que, en general, los surfistas buscan una vestimenta "para estar cómodo[s]". Esta identificación de estilo se extiende a cuestiones de carácter físico como "la marca del cuello", como indican el PPA3, el PPV5 o la PPC2. Otros aspectos físicos son los que ofrece la PPC6 cuando mantiene que, por ejemplo: "[...] el moreno de la cara... Tú le miras las manos, algo se puede

\footnotetext{
${ }^{4}$ El término "tubera" hace referencia a cuando las condiciones del mar presentan olas que forman un hueco entre el labio (parte superior de la ola) y la superficie en la que rompen originando un espacio que los surfistas denominan como "tubo" por la semejanza con la forma de ese hueco que forman,

5 TDA: Se refiere a un estilo que fluye. Por lo general, este anglicismo tiene connotaciones positivas.
} 
notar". En esta línea, algunos integrantes del grupo de Zarauz destacan que incluso es posible distinguir a unos surfistas expertos de otros que no lo son por otras cuestiones una vez que están dentro del agua. Según el PPV9, esto es posible reconocerlo "en cuestión de segundos" debido a la forma de remar, a las pegatinas que se llevan en la tabla, o, simplemente, al hecho de cómo coger la tabla. Estas apreciaciones, avanza, son cuestiones que raramente fallan a la hora de distinguir a los surfistas expertos de los iniciados. Lo que indica una de las características de la tribu como microgrupo, donde cada uno tiene su papel y su sitio dentro del mismo.

Por último, destaca que algunos de los términos que se emplean de manera reiterada en estas respuestas para referirse al conjunto de surfistas han sido las palabras tribu y subcultura, ambas cruciales para esta investigación. Algunos surfistas como el PPC10 se apoya en el término subcultura para concretar que los surfistas son "una subcultura. Ahora mismo es un grupo heterogéneo completamente, uno es policía, otro ingeniero otro tal, y llega del trabajo y viene del trabajo con una ropa y ... ¿no?". Para ejemplificar el uso del término tribu por parte de los entrevistados, es interesante atender a la apreciación del PPV2 quien señala que "[...] sí que debe haber conciencia colectiva a nivel de grupo, de tribu un poquito de, un poco casi a nivel de, no «postureo», pero como un poquito soy surfista". Igualmente, el PPG8 sugiere que entre los propios surfistas pueden existir muchas tribus: "Con toda la gente que surfea ahora, que es muchísima más que cuando nosotros nos conocimos al principio, ahora hay muchas, no me sale la esto, hay muchas tribus, muchas, no creo que se pueda ahora".

Con respecto a la distinción de un lenguaje o normas concretas, se observa que todos los participantes acuerdan reconocer que existe un "mínimo de idioma", "argot" o "jerga" que puede reconocerse entre los surfistas. En este contexto, algunas de las expresiones o palabras que han aparecido reiteradamente en las conversaciones han sido las que a continuación se muestran en la Tabla 2.

Tabla 2. Expresiones típicas del mundo del surf aparecidas durante la celebración de los focus groups. Fuente: Elaboración propia.

\begin{tabular}{|c|c|}
\hline Expresión & Explicación \\
\hline "Invento" & $\begin{array}{c}\text { Palabra utilizada para describir una cuerda flexible que } \\
\text { une la tabla con el tobillo y que evita que la propia } \\
\text { tabla se pierda }\end{array}$ \\
\hline "Pico" & Lugar en el mar donde rompen las olas \\
\hline "Hacer el pato o hacer la cuchara" & $\begin{array}{c}\text { Técnica que consiste en hundir la tabla a la vez que el } \\
\text { cuerpo para evitar que una ola te arrastre hasta la } \\
\text { orilla }\end{array}$ \\
\hline "La serie" & $\begin{array}{l}\text { Conjunto de olas que vienen de forma seguida una } \\
\text { detrás de otra }\end{array}$ \\
\hline "Hay un baño". & $\begin{array}{l}\text { Se refiere a que el mar tiene unas condiciones } \\
\text { óptimas para poder surfear }\end{array}$ \\
\hline “Está glassy” & $\begin{array}{c}\text { Se refiere a que no hay viento y por tanto, las olas } \\
\text { llegan de manera ordenada }\end{array}$ \\
\hline "Choppy" o "gocho" & $\begin{array}{l}\text { Se refiere a que hay viento y, por tanto, las olas no } \\
\text { Ilegan de manera ordenada }\end{array}$ \\
\hline "Está pasado" & $\begin{array}{c}\text { Se refiere a cuando las olas tienen un tamaño } \\
\text { demasiado grande como para poder surfear en una } \\
\text { playa determinada }\end{array}$ \\
\hline "Está plato" & $\begin{array}{l}\text { Se refiere a cuando en el mar no hay olas y se ve } \\
\text { completamente "plano" o "plato" }\end{array}$ \\
\hline
\end{tabular}




\begin{tabular}{c|c}
\hline "Mira esa izquierda, derecha" & $\begin{array}{c}\text { Se refiere a la denominación de olas que, bien pueden } \\
\text { partir hacia la izquierda, o bien, hacia la derecha } \\
\text { Movimiento del surfista en la tabla que suele dejar una } \\
\text { estela en la ola }\end{array}$ \\
\hline "Carving" & $\begin{array}{c}\text { Maniobra de surf que consiste en mantenerse sobre la } \\
\text { parte superior de la ola ya rota con la tabla de surf }\end{array}$ \\
\hline "Floater" & $\begin{array}{c}\text { Expresión utilizada para denominar a cualquier } \\
\text { maniobra en la que el surfista gire la tabla hacia algún } \\
\text { sentido }\end{array}$ \\
\hline "Gutback" & $\begin{array}{c}\text { Maniobra de surf que consiste en volver hacia atrás en } \\
\text { la ola para no perder velocidad }\end{array}$ \\
\hline "Reentry" & $\begin{array}{c}\text { Maniobra de surf que consiste en salir de la ola y } \\
\text { volver a entrar. Se trata de un giro complejo y rápido }\end{array}$ \\
\hline "Tubo" & $\begin{array}{c}\text { Maniobra de surf que consiste en introducirse dentro } \\
\text { de la ola una vez que ésta se encuentra rompiendo, } \\
\text { aprovechando así la forma tubular que adopta el agua }\end{array}$ \\
\hline
\end{tabular}

De hecho, destaca que el lenguaje empleado es algo tan característico de los surfistas que, en algunos casos, los sujetos sostienen que el conocimiento de estas palabras o expresiones puede llegar a diferenciar el nivel del mismo. Así lo entiende el PPG5, cuando comenta: "[...] a mí me pasaba más antes con algunos alumnos de iniciación de primer día que usas palabras que te parecen súper... Por ejemplo, dices pico, y ellos no saben qué es pico". En cuanto a la existencia de normas de surf, se ha observado que el conocimiento de ciertas normas es algo fundamental para la práctica revelándose que incluso su dominio puede desvelar el nivel del surfista.

En cuanto a la posibilidad de que los participantes reconocieran entre sus prácticas ciertos rituales, se ha encontrado, de nuevo, un amplio consenso entre los participantes. La totalidad de los surfistas reconoce llevar a cabo algún tipo de ritual durante la práctica del surf. En concreto, los entrevistados mantienen que la mayoría de los rituales tienen lugar antes de la práctica del surf. De entre estos rituales, destacan los indicados en la Tabla 3.

Tabla 3. Rituales de los surfistas participantes en los focus groups. Fuente: Elaboración propia.

\begin{tabular}{c|c|c|c}
\hline Tipo de ritual & Antes de la actividad & Durante la actividad & $\begin{array}{c}\text { Después de realizar } \\
\text { la actividad }\end{array}$ \\
\hline Calentar & $\mathrm{X}$ & & \\
\hline Encerado de la tabla & $\mathrm{X}$ & & \\
\hline $\begin{array}{c}\text { Evaluar las } \\
\text { condiciones del mar } \\
\text { de un vistazo }\end{array}$ & $\mathrm{X}$ & & \\
\hline $\begin{array}{c}\text { Colocarse el invento } \\
\text { Dar un beso al agua }\end{array}$ & $\mathrm{X}$ & & \\
\hline $\begin{array}{c}\text { Echarse agua en la } \\
\text { nuca }\end{array}$ & $\mathrm{X}$ & & \\
\hline Beber cerveza & $\mathrm{X}$ & $\mathrm{X}$ & \\
\hline $\begin{array}{c}\text { Colocación del } \\
\text { neopreno }\end{array}$ & & $\mathrm{X}$ & \\
\hline Remar & & & \\
\hline
\end{tabular}

${ }^{6}$ En la jerga surfista, este término hace referencia a la cuerda flexible que une la tabla de surf con el tobillo del surfista con el fin de evitar la pérdida de la misma. 


\begin{tabular}{c|c|c|c}
\hline $\begin{array}{c}\text { Ponerse de pie en la } \\
\text { tabla }\end{array}$ & & $\mathrm{X}$ & \\
\hline $\begin{array}{c}\text { Colocación de las } \\
\text { quillas en la tabla }\end{array}$ & $\mathrm{X}$ & & \\
\hline
\end{tabular}

Como indica la Tabla 3, existe un mayor número de rituales antes de iniciar la práctica del surf. En todos los focus groups destaca el acto de calentar y el encerado de la tabla. Al igual que ocurría en el caso del estilo, los sujetos subrayan que el modo de ejecución de estas prácticas puede indicar el nivel del surfista. Ejemplos como los del PPG9 así lo evidencian, cuando expresa que "es cruel, pero hasta el modo de llevar la tabla, tú ya sabes si esa persona surfea bien o no". De la misma manera lo expresa el PPV1 cuando asegura que: "Al poner la parafina tu puedes decir que ese lleva tres días o lleva toda su vida surfeando". En este sentido, el ritual parece no solo una práctica bien interiorizada, sino un modo de identificación entre los mismos y que va más allá de la mera práctica deportiva.

\section{CONCLUSIONES}

Estudiar el comportamiento de los sujetos desde un punto de vista tribal es una tarea que presenta notables complejidades. A pesar de que el interés académico por entender la forma en la que los sujetos se relacionan y agrupan es una tendencia destacable en la actualidad, se aprecia la necesidad de seguir investigando para aportar conocimientos demostrables que describan la realidad de los sujetos. En el caso concreto de este trabajo, se ha tratado de explicar el comportamiento de los surfistas desde una óptica tribal, algo que se ha podido comprobar gracias a la aplicación del diseño de esta investigación. En efecto, es posible afirmar que los surfistas españoles pueden englobarse en lo que se conoce como tribu urbana. Si bien de esta afirmación se pudiera desprender cierta obviedad, lo cierto es que los resultados obtenidos tras la aplicación de la metodología empleada para esa investigación, apoyan de manera clarificadora la idea de que el estudio de las tribus urbanas merece una mayor atención por parte de la academia desde puntos de vistas empíricos. Como se ha podido comprobar, a pesar de existir una gran cantidad de literatura académica que aborde los estudios de la tribu desde la perspectiva urbana y, de manera concreta desde un punto de vista teórico, los hallazgos presentados en este estudio ponen de manifiesto que es posible estudiar a estos grupos sociales desde una perspectiva que supere lo meramente teórico. Así lo demuestran los resultados obtenidos de los focus groups, donde se ha puesto de manifiesto que la práctica regular de rutinas y rituales que llevan a cabo estos individuos y la sacralización de espacios como la playa, no solo les permite y prepara para la correcta práctica del deporte, al mismo tiempo acerca a los sujetos provocando un sentimiento de pertenencia de grupo hacia sus miembros con los que se identifican y comparten pasiones comunes. También se ha podido observar cuestiones como el estilo. Uno de los rasgos que apoyan la caracterización de este grupo como tribu. Rasgos indentitarios concretos como la forma de vestir o incluso el aspecto físico, componen un ethos distintivo que ayuda a reconocer al otro como un igual en el que reflejarse y establecer relaciones que van más allá de lo exclusivamente deportivo. El empleo de ciertas expresiones y palabras describe también la caracterización de esta tribu. La manera de expresarse permite no solo la consecución eficaz de ciertos rituales y prácticas, asimismo permite experimentar a sus miembros la conciencia de grupo. Algo que, sin duda, se encuentra estrechamente relacionado con la evidencia de una serie de normas que comparten estos surfistas. Se ha observado que esta tribu interioriza una serie de pautas culturales que permiten adquirir ciertas destrezas y 
conocimientos que facilitan la práctica deportiva la vez que se configuran como patrones que facilitan la integración en el grupo.

En definitiva, los surfistas comparten una serie de rasgos claramente identificables que permiten su reconocimiento como una tribu concreta. No obstante, entre los hallazgos más significativos de esta investigación, es preciso destacar que el concepto de tribu urbana no solo es adecuado para describir las relaciones sociales establecidas entre jóvenes. Las edades comprendidas entre los entrevistados revelan que, en efecto, la pertenencia a este tipo de agrupaciones no ofrece ningún tipo de restricción etaria siendo un rasgo más del grupo. Esta cuestión pone de manifiesto la necesidad de abordar el concepto de tribu urbana desde una perspectiva renovada. Dado que la literatura disponible encuentra en la concepción de la tribu urbana condicionantes característicos como la edad o cierta afinidad con prácticas violentas, esta investigación revela que, en efecto, estos distintivos requieren de una consideración más amplia y aplicada según el grupo o segmento estudiado. De alguna manera, este estudio viene a plantear la posibilidad de redefinir dicho concepto con objeto de aplicarlo a grupos sociales avanzados que superen una limitación cultural o identitaria.

Como futuras líneas de investigación, sería interesante la realización de un nuevo estudio para identificar y describir a un mayor número de tribus con objeto de actualizar la literatura científica disponible. Asimismo, el marcado estilo que identifica a estas tribus puede abrir interesantes investigaciones acerca del estudio de estos sujetos desde perspectivas que superen lo meramente deportivo, abordando cuestiones como el consumo (tribus de consumo), el marketing, la socialización a través del deporte o los grupos emocionales vinculados a la práctica deportiva.

\section{REFERENCIAS}

Aguirre, A., y Rodríguez, M. (1997). Skins, Punkis, Okupas y otras tribus urbanas. Barcelona: Ediciones Bardenas.

Anderson, J. (2013). Cathedrals of the surf zone: regulating access to a space of spirituality. Social \& Cultural Geography, 14(8), 954-972.

Bauman, Z. (2010). Mundo consumo. Ética del individuo en la aldea global. Madrid: Espasa.

Booth, D. (1999). Surfing: The cultural and technological determinants of a dance. Culture, Sport, Society, 2(1), 36-55.

Booth, D. (2008). (Re)reading The Surfers' Bible: The affects of Tracks. Continuum, 22(1), 17-35.

Bourdieu, P. (1998). La distinción: criterios y bases sociales del gusto. Madrid: Taurus.

Caffarelli, C. (2008). Tribus urbanas, cazadoras de identidad. Buenos Aires: Lumen.

Colubi, P. (1997). El ritmo de las tribus. Barcelona: Alba Editorial.

Costa, P., Pérez, J. M., y Tropea, F. (1996). Tribus urbanas. El ansia de identidad juvenil: Entre el culto a la imagen y la autoafirmación a través de la violencia. Barcelona: Paidós. 
Esparza, D. (2016). Historia del surf en España. De Magallanes a los años 80. Olomouc: Edición Olo Surf History.

Esparza, D. (2019). Deporte femenino y tardofranquismo: las primeras surfistas en España. Materiales para la Historia del Deporte, 19, 61-77. Recuperado de https://www.upo.es/revistas/index.php/materiales historia deporte/article/view/ $\underline{3778}$

Feixa, C. (1999). De jóvenes, bandas y tribus. Barcelona: Ariel.

Fernández, C. (2000). Instrumentos de la psicología social para el análisis de los grupos. En A. Canteras, Sociología de grupos pequeños: sectas y tribus urbanas. Cuadernos de derecho judicial XI-2000 (pp. 267-319). Madrid: Consejo general del poder judicial.

Ganter, R., y Zarzuri, R. (1999). Tribus Urbanas: por el devenir cultural de nuevas sociabilidades juveniles. Revista de Trabajo Social Perspectivas, 8, 1-18.

Giner, S., Lamo de Espinosa, E. y Torres, C. (Eds.) (2006). Diccionario de Sociología. Madrid: Alianza.

Gonfaus, A. (2006). Surfers. Una visión antropológica del surf. San Sebastián: Zoe

Greco, O. (2003). Diccionario de sociología. Valletta Ediciones SRL.

Irwin, J. (1973). Surfing: The natural history of an urban scene. Urban life and culture, 2(2), 131-160.

Lefineau, M. (2010). Tribus urbanas: la indumentaria desde una perspectiva multicultural. Argentina: Nobuko.

Maffesoli, M. (1990). El tiempo de las tribus. Barcelona: ICARIA.

Márquez Lepe, E. (2015). Representaciones, identidades y nacionalismos en el discurso político español a propósito de la inmigración. En C. Sieber, V. Abrego y A. Burgert (Comps), Nación y Migración: España y Portugal frente a las migraciones contemporáneas (137-151). Madrid: Biblioteca Nueva.

Martín Criado, E. (1997). El grupo de discusión como situación social. Revista Española de Investigaciones Sociológicas, 79, 81-112.

Martín Criado, E. (1998). Producir la juventud. Crítica de la Sociología de la Juventud. Madrid: Ediciones Istmo S.L.

Matus, C. (2000). Tribus urbanas: entre ritos y consumos. El caso de la discoteque Blondie. Última Década, 13, 97-120.

Morgan, D. L. (1998). The Focus Group Guidebook. Thousand Oaks: Sage.

Moutinho, L., Dionísio, P., y Leal, C. (2007). Surf tribal behaviour: a sports marketing application. Marketing Intelligence \& Planning, 25(7), 668-690. 
Pellón, J. (2009). Guía del surf en España. Técnicas, playas surfeables, información turística y práctica y los mejores destinos internacionales. León: Destino Everest.

Preston-Whyte, R. (2002). Constructions of Surfing Space at Durban, South Africa. Tourism Geographies: An International Journal of Tourism Space, Place and Environment, 4(3), 307-328.

Rubin, H. J., y Rubin, I. S. (1995). Qulitative Interviewing. The Art of Hearing Data. Sage: Thousand Oaks.

Silva, J. (2002). “Juventud y tribus urbanas: en busca de la identidad. Última década, 10(17), 117-130.

Soto, M. L. (2012). Tribus urbanas y grupos indígenas: el imaginario social y la cultura como determinante en la concepción de la salud y la enfermedad. Méjico: Editorial Miguel Ángel Porrúa.

Stranger, M. (2010). Surface and substructure: beneath surfing's commodified surface. Sport in Society, 13(7), 1117-1134.

Tajfel, H. (1978). Differentiation between social groups: Studies in the social psychology of intergroup relations. London: Academic Press.

Taylor, S. J., Bogdan, R., y Devault, M. (2015). Introduction to qualitative research methods: A guidebook and resource. New Jersey: John Wiley \& Sons.

Turner, J. C., y Brown, R. (1978). Social status, cognitive alternatives and intergroup relations. En H. Tajfel (Ed.), Differentiation between social groups: Studies in the social psychology of intergroup relations (pp.201-234). Londres: Academic Press

Walle, A. H. (2015). Qualitative research in business: A practical Overview. New Castle: Cambridge Scholars Publishing.

Vargas, C. (2012). Culturas juveniles y tribus urbanas: ¿homogeneización o diferenciación? Praxis Pedagógica, 13,144-164.

Warren, A., y Gibson, C. (2017). Subcultural enterprises, brand value, and limits to financialized growth: The rise and fall of corporate surfing brands. Geoforum, 86, 177-187.

Zarzuri, R. (2000). Notas para una aproximación teórica a nuevas culturas juveniles: Las tribus urbanas. Última Década, 13, 81-96. 